\title{
Interview mit Ulrich Engel
}

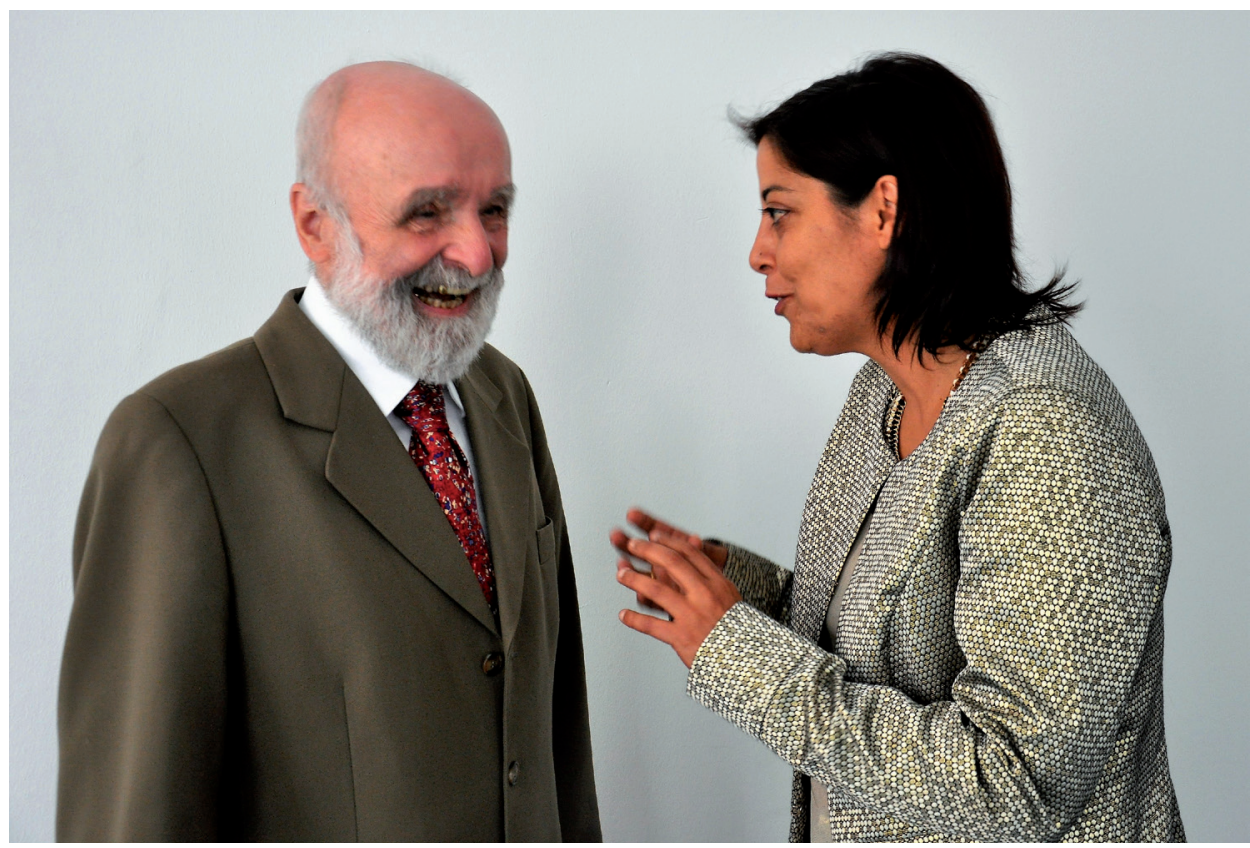

Prof. Dr. Dr. h. c. mult. Ulrich Engel und Prof. María José Domínguez Vázquez

„Von Nachbarn, Freunden, Kollegen, auch Genossen werde ich oft gefragt, womit ich mich eigentlich beruflich beschäftige. Ich sage dann im Allgemeinen, ich sei Sprachwissenschaftler. Auf diese Antwort erfolgen drei typische Reaktionen: Die Einen verlieren schlagartig das Interesse und wenden sich anderen Dingen zu, sie können mit mir einfach nichts mehr anfangen. Die Zweiten mimen schlagartiges Verständnis und fragen mich, wie ich zur Rechtschreibreform stehe, womit sie dem verbreiteten Unfug folgen, Sprache auf die Schreibung zu reduzieren. Die dritte, kleinste Menge aber ist erst einmal still, denkt also offenbar nach und fragt mich dann, wozu diese Wissenschaft wohl gut sei. Diese Reaktion ist durchaus verständlich: Ich weiß ja: Jeder Feuerwehrmann hat es leichter als ich, jeder Taxifahrer, jeder Bäcker, wenn er nach dem Sinn seines Berufes gefragt wird.

Ich möchte dies und jenes antworten, denn ich finde es schon faszinierend, sich mit der Fähigkeit, der einzigen, zu beschäftigen, die uns von allen Lebewesen unterscheidet.“ 
(Ulrich Engel, Rede bei der Verleihung der Ehrendoktorwürde an Ulrich Engel in Santiago de Compostela, am 8. Mai 2002, S. 11-12).

\section{Zur Person}

Ulrich Engel, geboren 1928 in Stuttgart, war Professor für Germanistik an der Universität Bonn. Er ist verheiratet und hat zwei Kinder und drei Enkelkinder.

Sein beruflicher Werdegang beginnt 1955 als Lehrer in Stuttgarter und Sindelfinger Gymnasien. Ein Jahr davor promovierte er an der Universität Tübingen mit der Arbeit Mundart und Umgangssprache in Württemberg. Zwölf Jahre lang war Ulrich Engel neben Paul Grebe Mitglied des Vorstands des Instituts für Deutsche Sprache in Mannheim, zunächst als Grebes Stellvertreter und seit 1970 als Direktor des IDS.

Ulrich Engel hat im Bereich der Dependenz- und Valenzgrammatik sowie der monolingualen und bilingualen Valenzlexikographie Pionierarbeit geleistet. Sein Ansatz beschreibt die auf hierarchischen Parametern aufbauende Anziehungskraft der Wörter, wobei dem Valenzträger ein entscheidendes Gewicht zukommt, denn er besitzt die Fähigkeit, Aktanten an sich zu binden. Zum Publikationsverzeichnis von Ulrich Engel gehören weltweit anerkannte Werke wie Deutsche Grammatik $\left({ }^{1} 1988,{ }^{3} 1996\right.$; Heidelberg) - unter Kollegen als ,die rote Bibel“" bekannt-, Kurze deutsche Grammatik (2002; München) und die Deutsche Grammatik - Neubearbeitung ( ${ }^{1} 2004,{ }^{2} 2009$; München), Syntax der deutschen Gegenwartssprache (1977, ${ }^{4} 2009$; Berlin) - in Valenzkreisen bekannt als ,der blaue Engel“". Unter den kontrastiven Grammatiken, die er geleitet bzw. an denen er als Autor mitgewirkt hat, sind zu nennen: Kontrastive Grammatik deutsch-serbokroatisch (1986; München), Kontrastive Grammatik deutsch-rumänisch (1993; Heidelberg), Kommunikative Grammatik. Deutsch als Fremdsprache (1993; München), Deutsch-polnische kontrastive Grammatik (1999; Heidelberg) und Deutsch-polnische kommunikative Grammatik (5 Bände, davon 4 erschienen). Die Entwicklung von Valenzwörterbüchern ist ebenfalls ein Leitfaden im Lebenslauf von Ulrich Engel. Er ist Mitverfasser folgender Valenzlexika: Valenzlexikon deutsch-rumänisch (1983; Heidelberg), Dictionar de verbe germane (2012 Iași), Wörterbuch zur Verbvalenz: Deutsch-Bosnisch/Kroatisch/Serbisch (2009; München), Wörterbuch zur Verbvalenz Serbisch-Deutsch (2013; München) und Valenzlexikon Deutsch-Spanisch (in Vorbereitung). Seine Valenzauffassung liegt außerdem zahlreichen Valenzlexika zugrunde, zum Beispiel dem Valenzwörterbuch deutscher und polnischer Verben (Morciniec/Cirko/Ziobro 1995; Wrocław), dem Valenzlexikon Deutsch-Italienisch (Bianco 1996; Heidelberg), dem Diccionario contrastivode valencias verbales espańolalemán (Rall/Rall/Zorrilla ${ }^{2} 1980$ Tübingen), dem Deutsch-Albanischen Valenzwörterbuch (in Vorbereitung), dem Deutsch-arabischen Valenzwörterbuch (in Vorbereitung) u. a., bei denen er als Berater mitgewirkt hat. Alle diese Werke sind durch Engels Genauigkeit bei der Beschreibung von häufig sehr komplizierten sprachlichen Phänomenen gekennzeichnet, vor allem durch Engels Fähigkeit, die Sprache akribisch und sorgfältig zu analysieren, insbesondere durch die Erläuterung des Komplizierten auf eine einfachere Weise. Sein wissenschaftlicher Lebenslauf ist damit aber noch nicht vollständig beschrieben. Hinzu kommen unzählige Aufsätze, die Leitung von Forschungsprojekten mit Bezug auf verschiedenste Sprachen in vielen Ländern sowie die Betreuung von Nachwuchswissen- 
schaftlern und Doktoranden aus der ganzen Welt. Hinter einer solchen Leistung muss Leidenschaft und Hingabe stehen.

\section{Interview}

D(ominguez): Lieber Herr Engel, an erster Stelle möchte ich mich bei Ihnen für Ihre Bereitschaft bedanken, sich von mir interviewen zu lassen.

E(ngel): Ach Gott - wenn mich Leute, die ich ernst nehme, auf seriöse Weise fragen, werde ich immer antworten.

D: Zu Beginn möchte ich ein wenig in die Vergangenheit zurückblicken. Was war für Sie das Positivste in Ihrer Zeit als Gymnasiallehrer? Vermissen Sie etwas aus dieser Zeit?

E: Das Wichtigste und das Schönste war in meiner Lehrerzeit der Kontakt mit den Schülern, das Zweite die Aufarbeitung jüngster deutscher Vergangenheit (ich gab unter anderem Geschichtsunterricht). Da war viel Überzeugungsarbeit zu leisten. Sie müssen sich vorstellen: Dort war eine Bundeswehrkaserne, viele Schüler waren Offiziers- oder Soldatenkinder, die kamen mit eigenen Vorstellungen wie „War doch alles nicht so schlimm“ oder gar „Sollen wir denn das eigene Nest beschmutzen?“ Diese Zeit war hart, und sie war gut, für mich und die jungen Leute. Eine Klasse lädt mich heute noch zu ihren Treffen ein, mit einigen der ehemaligen Schüler bin ich befreundet. Diese Kontakte mit Schülerinnen und Schülern vermisse ich - sonst nichts.

D: Wann bzw. warum haben Sie sich für die Lehre und Forschung an der Universität entschieden? Welche Faktoren haben dabei eine Rolle gespielt?

E: Eigentlich wurde ich geschoben. Ich wollte zunächst immer Lehrer bleiben, das machte mir Freude und ich sah einen Sinn darin. Es war Hugo Moser, der mich unbedingt an der Universität haben wollte und mich schließlich dazu brachte, dass ich ein dreijähriges Habilitandenstipendium annahm. Mein Thema war die „deutsche Alltagssprache“. Am Ende ging ich gerne wieder in den Schuldienst zurück.

Hier allerdings wurde mir schnell klar, dass dieser erneute Wechsel einen geistigen Abstieg mit sich brachte. Die Lehrerkollegen sorgten sich vor allem um ihre Beamtengehälter, die meisten waren für wirklich Wichtiges nicht zu gewinnen. Seither suchte ich nach einem Platz in der Forschung. Der entscheidende Moment war die Dudenpreis-Verleihung an Hugo Moser (1964). Bei dieser Gelegenheit wurde auch die Gründung des Instituts für deutsche Sprache bekannt gegeben. Ich sagte ihm, dass mich das interessieren würde, und dachte eigentlich nur an eine Mitarbeit als Wissenschaftler. Aber das Kuratorium entschied dann auf seinen Vorschlag hin, dass ich Vorstandsmitglied werden solle, und das war mir dann eben auch recht.

D: Lieber Herr Engel, Sie erzählen immer, dass Sie auf die Valenzgrammatik durch Zufall gestoßen sind und nennen in diesem Zusammenhang Tesniére. Könnten Sie mir bitte erklären, was an Tesniéres Werken ihr Interesse geweckt hat, konkret: Warum die Dependenz- und Valenzgrammatik? Was begeistert Sie an der Valenzgrammatik?

E: Das ist ein bisschen viel auf einmal. Zuerst Tesniére: Ich wurde in Leo Weisgerbers Arbeitskreis „Sprache und Gesellschaft“ von verschiedenen Leuten auf ihn hingewiesen, informierte mich zunächst oberflächlich, las dann aber Erbens „Abriss der deutschen Grammatik“ von 1958, der zwar Tesniéres Hauptwerk damals nicht kannte, aber nahezu 
identische Ideen umsetzte. Ich las dann Tesniére während eines Krankenhausaufenthalts und fand ihn in seinen Hauptvorstellungen überzeugend. Das ist eigentlich alles. In meiner IDS-Zeit unterwarf ich mich nicht der damals herrschenden Mode, der Generativen Transformationsgrammatik zu folgen, las zwar sehr eingehend Chomskys "Aspects“ von 1965, fand auch, dass die GTG in ihrem Basisteil, der ja auch heute noch das Ganze prägt, ungemein traditionell geblieben war, und das gilt bis auf den heutigen Tag. Tesniére fand ich da wesentlich überzeugender. Ich bin nicht „begeistert“ von der Dependenz- und Valenztheorie, halte sie aber für fähig, alle sprachlichen Erscheinungen zureichend $\mathrm{zu}$ beschreiben, und ich finde sie, vor allem natürlich in neueren Ausprägungen, in ihrer Satzgliedsystematik besser als alle anderen Verfahren. Und ich halte es für einen Vorteil, dass unsere Dependenzgrammatik immer von den vorhandenen Elementen ausgeht und, indem sie sie zueinander in Beziehung setzt, aus ihnen „höhere“ Kategorien ableitet.

D: Was hat Sie dazu bewegt, so eine anspruchsvolle und breit angelegte Grammatik wie Ihre Deutsche Grammatik zu schreiben? Warum überhaupt eine Grammatik? Woher kamen die Anstöße? Und wie plant man so ein umfangreiches Werk?

E: Nun zu meiner Grammatik. Das war, wie übrigens vieles in meinem Leben, kein Plan, den ich mir gesetzt hatte; der Anstoß kam vielmehr von außen: Der Klett-Verlag wollte damals von mir eine „Deutsche Grammatik“. Es ist eine andere, hier nicht relevante Geschichte, warum das Buch dann bei Groos in Heidelberg und die Neubearbeitung bei iudicium in München erschien. Das Buch hat jetzt insgesamt fünf Auflagen erlebt, also wird es offenbar gebraucht.

D: Es kann nicht anders sein - ich möchte Ihnen einige Fragen zum Kern Ihrer Theorie, zu der Dependenz- und Valenzgrammatik, stellen: Warum fassen Sie das Verb als Valenzträger, als Zentrum des Satzes, auf?

E: Das geht auf Tesniére zurück. Mir leuchtete ein, dass vom Verb wichtige Satzglieder, die wir Ergänzungen nennen, selegiert werden und damit eine Minimalstruktur des Satzes festgelegt wird. Dieses Faktum ist so überzeugend, dass es auch von der IDS-Grammatik (1997), keiner Dependenzgrammatik, übernommen wurde.

D: $\mathrm{Ab}$ und an wird aufgrund der Schwierigkeiten zur Feststellung einer sauberen Grenze zwischen Ergänzungen und Angaben - die als Grundsäule jeder valenzbasierten Analyse aufzufassen ist - die Valenzgrammatik als wacklig bzw. als sich nichtaufrechtzuhaltender sprachwissenschaftlicher Ansatz beschrieben. Wie wird in Ihrem Ansatz diese Abgrenzung erklärt?

E: Ergänzungen sind, wie Sie wissen, nur von einer Subklasse von Verben zugelassen oder gefordert, somit nicht bei allen Verben möglich; vergleichen Sie dazu die subjektlosen Sätze wie Es regnet., wo es allein vom Verb abhängt, dass hier kein Subjekt möglich ist. Ergänzungen sind also subklassenspezifische Elemente, Angaben dagegen unspezifisch, weil sie bei jedem Verb vorkommen können. Diese Unterscheidung halte ich für klar und einfach. Alle Ansätze, die Klarheit dieser Unterscheidung zu bezweifeln, beruhen auf Missverständnissen.

D: Und warum ist das Thema der freien Dative sowie der Valenzerweiterungen immer noch so aktuell? Warum werden sie häufig als Beispiel für eine Schwachstelle der Valenz- und Dependenzgrammatik angeführt?

E: Das ist ein weiteres Beispiel für irrtümliche, weil oberflächliche Argumentation. Es handelt sich, ich muss da ein wenig ausholen, um den dativus possessivus, sympathicus, incommodi und ethicus. Zwei davon (sympathicus und incommodi) sind subklas- 
senspezifisch, mithin Ergänzungen, sie kommen also nur bei bestimmten Verben vor. Der sympathicus (Sie hat mir den Ärmel geflickt.) ist nur bei Verben möglich, die ein willkürliches Handeln bezeichnen. Der incommodi (Das Glas ist mir runtergefallen.) ist auf bestimmte Vorgangsverben beschränkt. Der dativus possessivus bildet eine Ausnahme: Er ist nicht verbabhängig, sondern Attribut in einem Satzglied: Das mir in Der Hund hat mir den Ärmel zerrissen. hängt von dem Nomen Ärmel ab und kann nur bei Körperteilen, Kleidungsstucken und Ähnlichem vorkommen.

Der (immer pronominale) ethicus (Gib Acht, sonst fällst du mir noch runter.) ist wohl als Angabe aufzufassen. Er drückt persönliches Engagement, Betroffensein des Sprechers oder des Hörers aus und ist, soweit absehbar, bei beliebigen Verben möglich. Er kann also, wenn „Freiheit“ Aspezifizität bedeutet, als Einziger „frei“ genannt werden.

Wer aber alle diese Dative pauschal als frei bezeichnet, der hat entweder keine Ahnung von Valenz - oder er hat einfach nicht aufgepasst.

D: Noch eine Frage zu dem Untersuchungsgegenstand der Valenz. Was kann die Valenzgrammatik nicht beschreiben? Was hat sie nicht als Untersuchungsgegenstand?

E: Wir müssen unterscheiden: Wenn man unter „Valenzgrammatik“ das versteht, was heute neben uns viele Leute in Deutschland und auch in anderen Ländern betreiben, dann bleibt der Anspruch, dass sie letzten Endes alle sprachlichen Phänomene beschreiben und erklären und alle sprachlichen Probleme lösen kann.

Wenn Sie aber nach den Möglichkeiten der Valenz fragen, so muss gesagt werden, dass sie eine Teilkategorie der Dependenz ist. Dependenz wiederum ist eine graphische Version der Konkomitanz, also der wechselseitigen Vorkommensbedingungen sprachlicher Elemente: Ein Element, das das Vorkommen anderer Elemente steuert, ist diesen übergeordnet, regiert sie also. Und die Valenz selegiert unter den abhängigen Elementen eben die subklassenspezifischen. Insofern gibt es natürlich ungeheuer viele Phänomene, die die Valenz nicht beschreiben, bzw. Probleme, die sie nicht lösen kann. Valenz ist eine kasuelle Eigenschaft der Dependenz, sie beschreibt spezifische Abhängigkeiten. Mehr kann sie nicht, mehr will sie nicht.

D: In Ihrer Rede als Ehrendoktor der Universität Santiago de Compostela haben Sie mit einer sehr schonen Bezugnahme auf die Stadt und ihre Bedeutung als Pilgerziel die Lage der Dependenzgrammatik sowie ihrer Anhänger beschrieben, und zwar:

„Unter diesen streitbaren Wissenschaftlern, die alle auf je eigene Art die Dependenzgrammatik vertreten, sollte man sich nichts Falsches vorstellen. Wir sind, ein gutes Dutzend, kein verschworener Orden, der nur seinen heiligen Gral hütet und alle Andersdenkenden bekämpft. Wir sind so etwas wie Pilger, die, aus verschiedenen geistigen Landschaften, einem Ziel zustreben.“(17-18).

Da wir nur ein gutes Dutzend sind, frage ich mich, wie Sie sich die Zukunft der Dependenz und Valenzgrammatik vorstellen. Wohin steuert die Valenzgrammatik? Was ist in diesem Bereich noch zu machen? Was ist noch nicht gemacht worden? Warum?

E: Also erstmal: Wir sind heute erheblich mehr als ein Dutzend. Zwischen den einzelnen Dependenzlern gibt es zwar Unterschiede, aber die greifen nie an die Basis. Wohin die Valenzgrammatik steuert? Darüber habe ich 2002 in Santiago de Compostela mit Eroms diskutiert. Dazu kann ich heute nicht mehr sagen als damals. Zu tun, meine Güte, ist noch unendlich viel. Wir müssen Verbgruppen untersuchen, wir müssen die Valenz von Nomina und Adjektiven noch besser eruieren. Wir müssen den Angaben mehr Aufmerksamkeit schenken. Und wir müssen überlegen, wie das Dependenzprinzip - und damit 
auch das Valenzprinzip - vom Satz auf den Text übertragen werden kann. Es ist noch unendlich viel zu tun. Aber das gilt für alle Theorien.

D: Soll ich daraus schließen, dass Sie sich damit noch beschäftigen werden, oder wie geht es jetzt weiter? Was bleibt Ihnen noch zu tun?

E: Wir können, Sie und ich, gar nichts schließen. Das menschliche Leben stößt irgendwo an Grenzen. Wie lange ich noch weitermachen kann, steht in den Sternen. Mindestens so viel kann ich sagen: Im Augenblick sieht es recht gut aus. Aber was ich künftig machen werde? Ich will meine Projekte zu Ende bringen: eine fünfbändige deutsch-serbische kontrastive Grammatik, eine fünfbändige deutsch-polnische kommunikative Grammatik, die zweite Auflage der deutsch-polnischen kontrastiven Grammatik, ja - und unser gemeinsames Projekt, das deutsch-spanische Valenzlexikon, dessen zwei Bände gerade in den Druck gehen. Und ich will denen, die auf der Basis meiner Grammatik Detailprojekte vorantreiben, Valenzlexika meistens, helfen, so lange ich noch kann. Reicht das?

D: Ich möchte Ihnen noch einige generelle Fragen stellen: Welches ist der größte Unfug, den Sie im Bereich der Sprachwissenschaft gehört oder gelesen haben?

E: Ich weiß nicht recht, da ist so viel passiert. Wahrscheinlich der Versuch, Zweisprachigkeit als charaktergefährdend zu erweisen (in der Hitlerzeit). Oder der Versuch, die GTG unmittelbar in ein Schullehrbuch umzusetzen.

D: Und etwas was Sie geschrieben haben und heute bereuen?

E: Nein. Ich habe einiges geschrieben, was ich heute anders schreiben würde. Ich sehe aber nichts, was ich bereuen sollte.

D: Was hätten Sie heute anders gemacht?

E: Ich hätte mich nicht, als Direktor des IDS, für wissenschaftsfremde Aufgaben einspannen lassen sollen. Das können andere besser (und machen es lieber) als ich. Das heißt: Ich hatte mich mehr und ausschließlich der Linguistik widmen sollen. Könnte ich noch einmal anfangen, so wäre das mein Leitstern.

D: Herr Engel, wenn Sie das erlauben, würde ich Ihnen gerne zum Spaß drei Fragen stellen, die bestimmt in keinem Buch bzw. Interview von Ihnen beantwortet worden sind: Könnten Sie zwei positive und zwei negative Eigenschaften von Ihnen nennen?

E: Positiv: ich singe gern; ich lache gern (aber nur wenn ein geeigneter Anlass vorhanden ist). Negativ: Ich habe keine Freude am Essen; ich kenne fast keine Pflanzen, Gänseblümchen und Löwenzahn schon, aber nicht viel mehr.

D: Welches ist ihr Lieblingslied und -buch?

E: Lieblingslieder habe ich zahllose. Im Augenblick fallen mir zwei ein: Das Lied Ich möchte so gerne hüpfen, das meine Frau mit den Kindern sang, als sie noch Elternund-Kind-Turnen gab. Das andere ist das Studentenlied Im tiefen Keller sitz ich hier, nicht wegen des schrecklichen Textes, sondern wegen der eindrucksvollen Melodie, die zwei Oktaven umfasst. Ich kann es leider nicht mehr singen, ich schaffe mit Mühe noch eine Oktave und eine Sexte.

$\mathrm{Zu}$ den Büchern: Mein Lieblingsbuch ist immer noch das Nibelungenlied, dieses scheußliche Drama von Totschlägern, und das alles wegen zweier Frauen. Die Dichtung enthält aber auch eine große Zahl wunderschöner Schilderungen. Ebenso lieb ist mir ein Buch mit Rilkes Gedichten. Wenn Sie mich aber im nächsten Jahr zu Liedern und Büchern befragen, kriegen Sie möglicherweise andere Antworten.

D: Was machen Sie in Ihrer Freizeit, falls Sie sie haben?

E: Gartenarbeit. 
D: Herr Engel, vielen Dank für diese angenehme Zeit und für Ihre Bereitschaft zu antworten. Ich wünsche Ihnen viel Erfolg bei den von Ihnen noch zu vollendenden Forschungsprojekten sowie viel Freude an der Arbeit und am Leben.

E: Danke. Ich wünsche Ihnen dasselbe, und bei Ihnen ist es ja wesentlich wichtiger, weil Sie noch vieles werden bewältigen können.

Es war, wie immer, ein Vergnügen, sich mit Ulrich Engel zu unterhalten. Etwas kann Engel nicht abgesprochen werden: seine Begeisterung für Phänomene aus den unterschiedlichen Sprachen sowie seine Offenheit und Bereitschaft, sich damit zu beschäftigen. Jeder, der Rat sucht, findet bei Ulrich Engel Unterstützung und vielfältige Antworten. Ulrich Engel ist stets engagiert, sowohl in beruflicher wie in privater Hinsicht - privat mit der unschätzbaren Hilfe seiner Frau. Das, meine Damen und Herren, ist heutzutage, wo alles viel Zeit kostet, besonders hervorzuheben. Und dies haben die zahlreichen Kollegen und Freunde aus der ganzen Welt an ihm geschätzt: Nicht umsonst ist Ulrich Engel Ehrendoktor der Universität Breslau/Wrocław, der Universität Santiago de Compostela, der Universität Bukarest und der Universität Hermannstadt/Sibiu. Er hat zudem viele Preise erhalten und ihm sind mehrere Ehrenmonographien gewidmet worden.

María José Domínguez Vázquez Universidade de Santiago de Compostela, Spanien

\section{Literatur}

ENGEL Ulrich, 2002, Discursos da investidura de D. Ulrich Engel como Doutor „Honoris Causa“: [Verleihung der Ehrendoktorwurde an Herrn Prof. Dr. Ulrich Engel], Universidad de Santiago de Compostela, Santiago de Compostela. 